\title{
Contrast sensitivity versus visual acuity in retinal disease
}

\author{
MICHAEL F MARMOR
}

From the Division of Ophthalmology, Stanford University, and the Ophthalmology Section, Veterans Administration Medical Center, Palo Alto, CA, USA

SUMMARY A large group of individuals with retinal disease were tested prospectively for contrast sensitivity by means of Arden gratings. A subgroup of 19 were also tested with the Nicolet automated television system. Individuals with macular or peripheral dystrophy showed a general reduction in contrast sensitivity as visual acuity decreased. The loss of contrast sensitivity was more prominent for high spatial frequencies $(6 \cdot 4$ cycles per degree) than for low ones $(0 \cdot 2$ cycles per degree). Similar results were obtained for patients with achromatopsia and congenital stationary night blindness. Patients with functional complaints, but no organic basis for decreased acuity, showed greater scatter in their test scores. The Nicolet results showed somewhat smoother curves, but were no more specific in separating normality from abnormality. Contrast sensitivity testing was not specific for the retinal disease entities considered, but may be useful in recording a degree of retinal damage and a degree of functional visual disability. Contrast sensitivity appeared to be reduced whenever acuity was reduced, so that a distinction could be made between patients having a loss of contrast beyond the expectations for their level of acuity and those in whom a loss of contrast simply corroborates the reduction of acuity.

Snellen visual acuity testing is a relatively crude index of visual function, in so far as it measures resolution only with high contrast targets, since objects in the real world exhibit varying degrees of contrast and a varying content of spatial frequencies. There has been considerable interest in recent years in developing practical clinical tests for contrast sensitivity. Several systems are now commercially available, including a set of printed plates, ${ }^{12}$ a computerised television system, ${ }^{3}$ and a set of posters. ${ }^{4}$ Reports have appeared showing that contrast sensitivity deficits out of proportion to visual acuity may be found in patients with retinal or optic nerve disease. ${ }^{25-10}$ There have been no large series published, however, to show the incidence of contrast sensitivity defects in macular or retinal disease, or to show whether the pattern of loss has diagnostic or prognostic value. There is also little information on what levels of contrast deficit to expect simply on the basis of decreased acuity. The present study shows the result of prospective contrast sensitivity testing with Arden

Correspondence to Michael F Marmor, MD, Division of Ophthalmology, Stanford University Medical Center, Stanford, CA 94305, USA. gratings on a large group of patients and on a subgroup with the Nicolet television system.

\section{Material and methods}

Over a two-year period all patients referred for clinical visual electrophysiological testing at the Stanford University Medical Center were given the Arden grating test. All testing was performed by a single examiner, who followed the technique of Arden et al.$^{12}$ Each grating was covered with a sheet of grey paper that was slowly removed, exposing progressively higher levels of contrast. The six vertical sinusoidal gratings (numbered 2-7) have spatial frequencies of $0 \cdot 2,0 \cdot 4,0 \cdot 8,1 \cdot 6,3 \cdot 2$, and $6 \cdot 4$ cycles per degree (cpd) respectively. Marginal numbers range from 0 at the low contrast end to 20 at the high contrast end; a score of 25 is assigned arbitrarily if the subject is unable to perceive the grating.

For a period of three months we had use of an Optronix 2000 contrast sensitivity test system, courtesy of Nicolet Instrument Company. We programmed this system to mimic the Arden test protocol: gratings of $0.5,1 \cdot 5,3 \cdot 0$, and $5.71 \mathrm{cpd}$ were 
presented by the 'method of increasing contrast', which requires the observer to push a button when the grating first becomes visible. The pattern was presented four times at each spatial frequency.

We have tabulated data from subjects who fit into several major diagnostic categories. 'Normals' were 16 persons aged $10-33$ with $20 / 20$ acuity, no symptoms, and no evidence of ocular disease; most of them were unaffected family members of patients with disease. Patients with 'macular dystrophy' $(\mathrm{n}=$ 50 ), aged 6-76, included some with Stargardt's disease, Best's vitelliform dystrophy, and patients with unclassified bilateral maculopathy that could not be ascribed to infectious, degenerative, or other secondary causes. Patients with 'diffuse dystrophy' $(n=61$, ages 6-66) include those with retinitis pigmentosa and its variants, as well as unclassified conditions in which there was bilateral diffuse retinal damage noted by ERG or fundus examination, and no evidence for an acquired aetiology. Specifically excluded from this group are carriers of X-linked retinitis pigmentosa, individuals with mild or 'delimited' forms of retinitis pigmentosa, " and patients with predominantly cone or central dystrophies. Patients with achromatopsia $(n=6$, ages $10-34)$ and congenital stationary night blindness $(n=7$, ages 8-47) were classified on the basis of ERGs that showed an absence of cone or rod function respectively, and had histories and physical findings consistent with stationary congenital disease. Patients with 'functional complaints' $(n=19$, ages 8-66) had visual symptoms that could not be substantiated by physical findings.

Snellen visual acuities were tested with a conventional clinical projection system using the subjects' best known correction. However, many of the subjects were referred only for electrophysiological testing, and the accuracy of their refraction was not confirmed.

\section{Results}

Arden grating scores for normal subjects (one eye only) with $20 / 20$ vision were close to Arden's values. ${ }^{1}$ The total score (sum of all six gratings) averaged $65 \cdot 0 \pm 6 \cdot 8$ (standard deviation). Despite the manual technique for unmasking the gratings, Arden scores were remarkably consistent. Four patients with stable disease had repeat testing at intervals between 7 and 21 months, and their total scores did not vary more than $10 \%$.

Fig. 1 shows data from all eyes with macular dystrophy. The total Arden grating scores (top) generally increased as visual acuity decreased, although a wide range of total scores are evident even among those eyes with good acuity (20/40 or better).
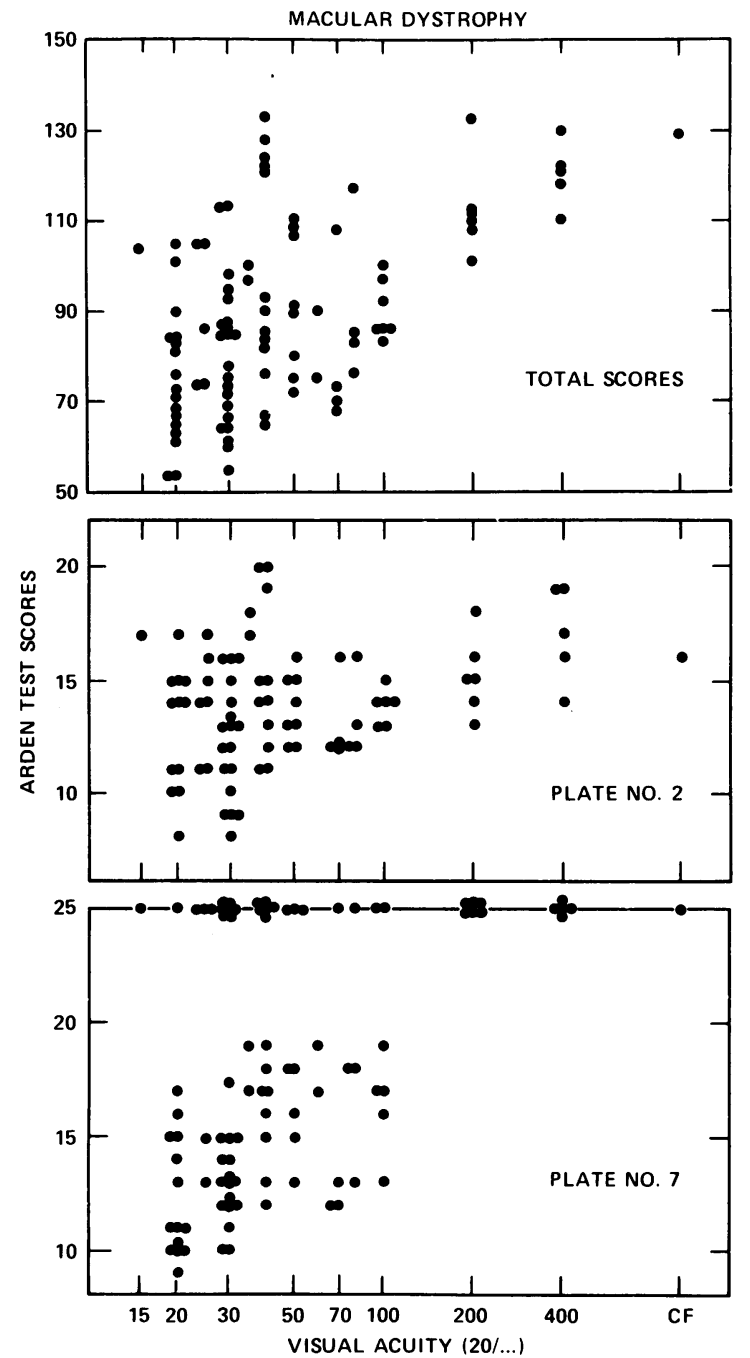

Fig. 1 Arden grating scores from eyes with macular dystrophy. The data are plotted against a logarithmic scale of visual acuity. Top: Total scores. Middle: Scores on plate number $2(0 \cdot 2 \mathrm{cpd})$. Bottom: Scores on plate number 7 (6.4 cpd).

The middle and bottom parts of Fig. 2 show scores from the coarsest $(0.2 \mathrm{cpd})$ and finest $(6.4 \mathrm{cpd})$ gratings respectively. All of the eyes with macular dystrophy could perceive the coarse grating, but a significant number of eyes could not appreciate the finest grating (scores of 25 ) even though acuity was excellent.

Similar results were obtained from patients with diffuse dystrophies (Fig. 2).

Fig. 3 shows the results from patients with achromatopsia and congenital stationary night blindness (CSNB), both non-progressive disorders of retinal 

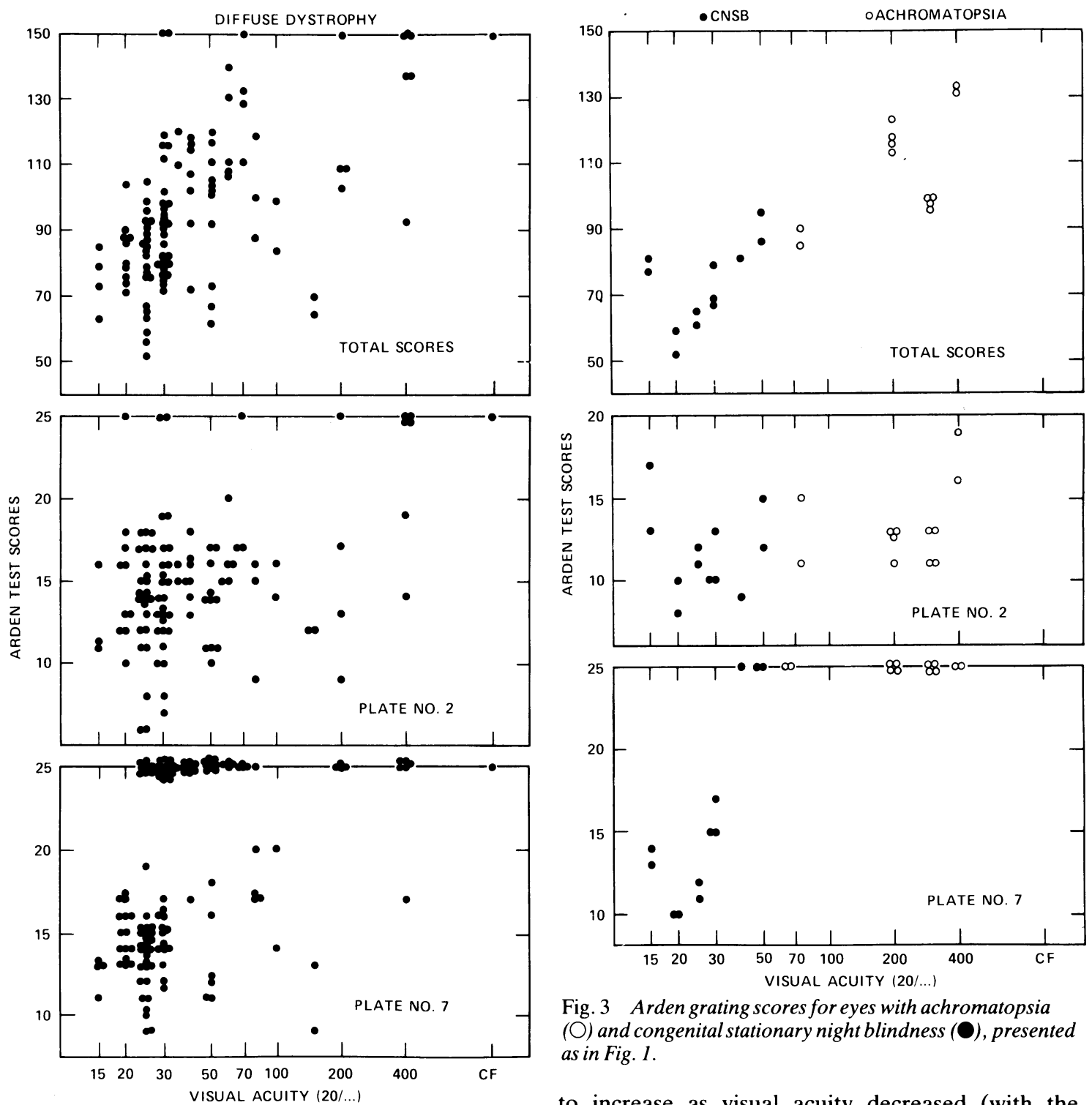

Fig. 2 Arden grating scores from eyes with diffuse dystrophy, presented as in Fig. 1.

function. The total scores generally correlated with the levels of acuity in these subjects. All subjects perceived the coarsest grating, but those with reduced acuity could not perceive the finest one.

The patients with 'functional complaints' (Fig. 4) had a range of symptoms including poor acuity, sensitivity to glare, poor dark adaptation, and field constriction, but they showed no clear fundus abnormalities and had normal electrophysiological test results. The total grating scores for their eyes tended

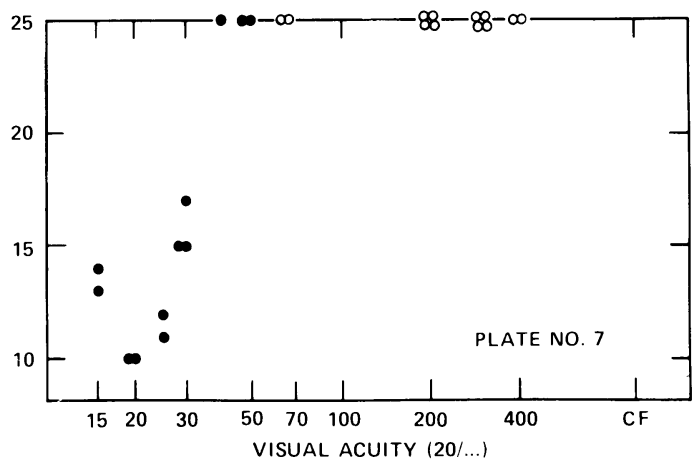

Fig. 3 Arden grating scores for eyes with achromatopsia (O) and congenital stationary night blindness $(\mathbf{O})$, presented as in Fig. 1.

to increase as visual acuity decreased (with the exception of two questionably low scores in a patient who tested at 20/70 acuity), but the scatter was more pronounced and less predictable for each individual grating.

Fig. 5 compares results obtained with Arden gratings with those obtained with the Nicolet unit at similar spatial frequencies and with a similar test protocol (method of increasing contrast). Because of the different clinical scoring methods the best performances shown in Fig. 5 lie near the bottom of the Arden graphs but near the top of the Nicolet graphs. We did not have the opportunity to obtain normative control data for the Nicolet unit, and the 'normal' 

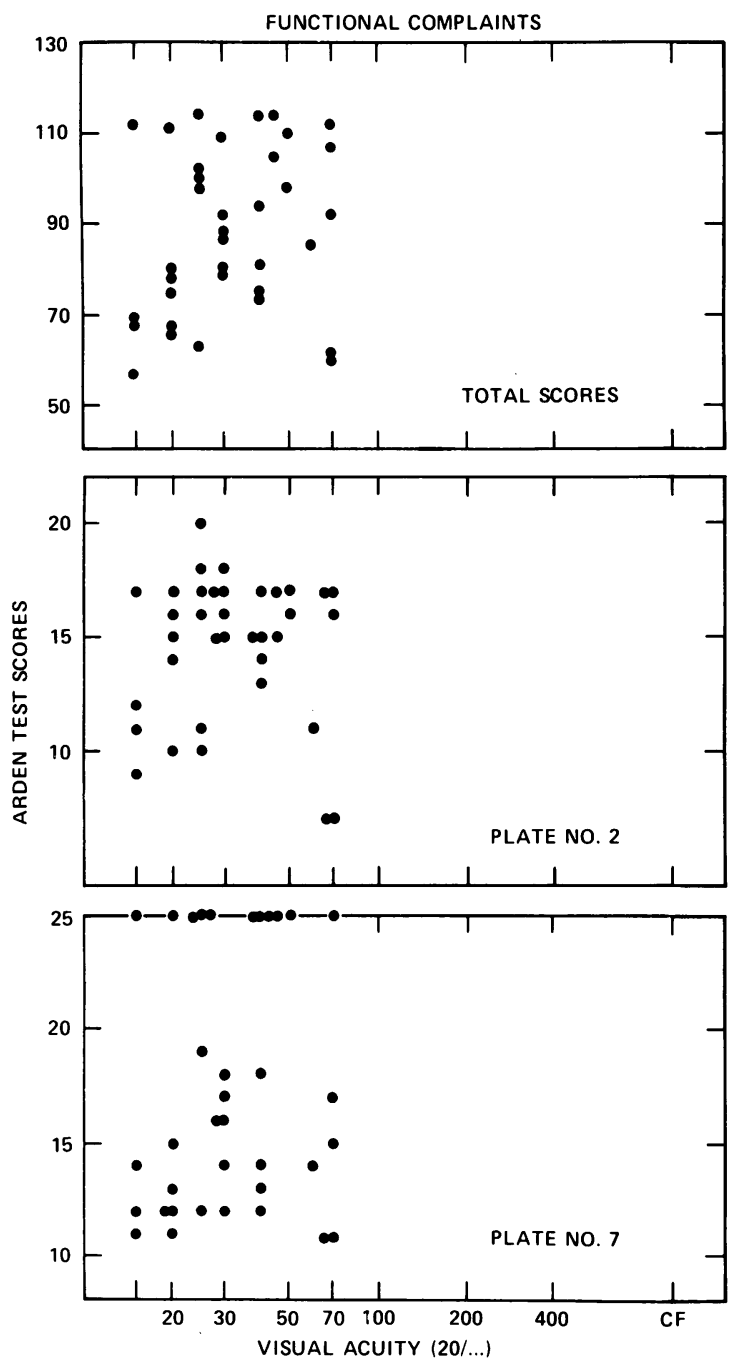

Fig. 4 Arden grating scores for patients with functional visual complaints but no demonstrable organic disease.

values shown were copied from the literature. ${ }^{3}$ They may well be too high, since they were obtained using the von Bekesy test protocol, which tends to yield higher sensitivity values than the method of increasing contrast. ${ }^{12}$

Of the subjects with macular dystrophy (Fig. 5, top), those with relatively good acuity showed relatively normal contrast sensitivity functions, measured by either system. Since the true range of normal for the Nicolet results is probably lower than illustrated, it may well incorporate all of the top four curves in the upper right panel. The two individuals $(\square, \Delta)$ with very poor contrast sensitivity (visual acuities $20 / 60,20 / 100$ ) were abnormal by both tests. There was somewhat greater scatter of data with the Arden gratings, but the Arden gratings also identified some abnormal responses to (e.g., to $6.4 \mathrm{cpd}$ for subjects and $O$ ) that were not clearly evident on the Nicolet testing. The patients with diffuse dystrophy (Fig. 5, middle) showed, by either technique, a greater loss of high than low frequency sensitivity. The Arden results showed a severely abnormal contrast sensitivity for four of the six eyes, whereas only three of the six were severely abnormal by Nicolet testing. The patients with functional complaints (Fig. 5, bottom) showed considerable scatter in their Arden results and had scores that sometimes fell in the borderline or elevated range. On testing with the Nicolet unit they showed more consistent curves, with predominant loss of sensitivity at higher frequencies.

\section{Discussion}

These data show clearly that contrast sensitivity is often subnormal in patients with retinal disease who still have relatively good acuity as measured on the Snellen chart. Conversely, some eyes with reduced central vision may show surprisingly good contrast sensitivity, especially for gratings of low spatial frequency. These general conclusions are consistent with earlier studies ${ }^{s-10}$ and show as well that patterns of contrast deficiency are not very specific to one or another type of ocular pathology. For all of the retinal diseases tested the contrast sensitivity loss was most severe at higher spatial frequencies, and low frequencies were rarely compromised unless there was also a severe loss of visual acuity. The patients with functional complaints showed a scatter of data on contrast sensitivity testing, but this is consistent with their variable performance on other subjective tests.

These results do not mean that contrast sensitivity testing is of no value, of course. Contrast sensitivity testing may detect early organic damage before acuity is compromised; it may also help to follow (with coarse gratings) the progression of severe disease when acuity is already at a low level. Furthermore, the assessment of functional disability gives us understanding of how well adapted or badly adapted our patients are to visual tasks in the real world.

The Arden gratings have been criticised because of the manual procedure for uncovering the gratings. Arden $^{12}$ has claimed that the errors produced by operator variability are relatively minor, and this was our experience as well. The automated Nicolet system produced smoother curves than the Arden plates, but it did not appear any easier to separate normality from abnormality with the Nicolet data.

This study points out the importance of interpreting 

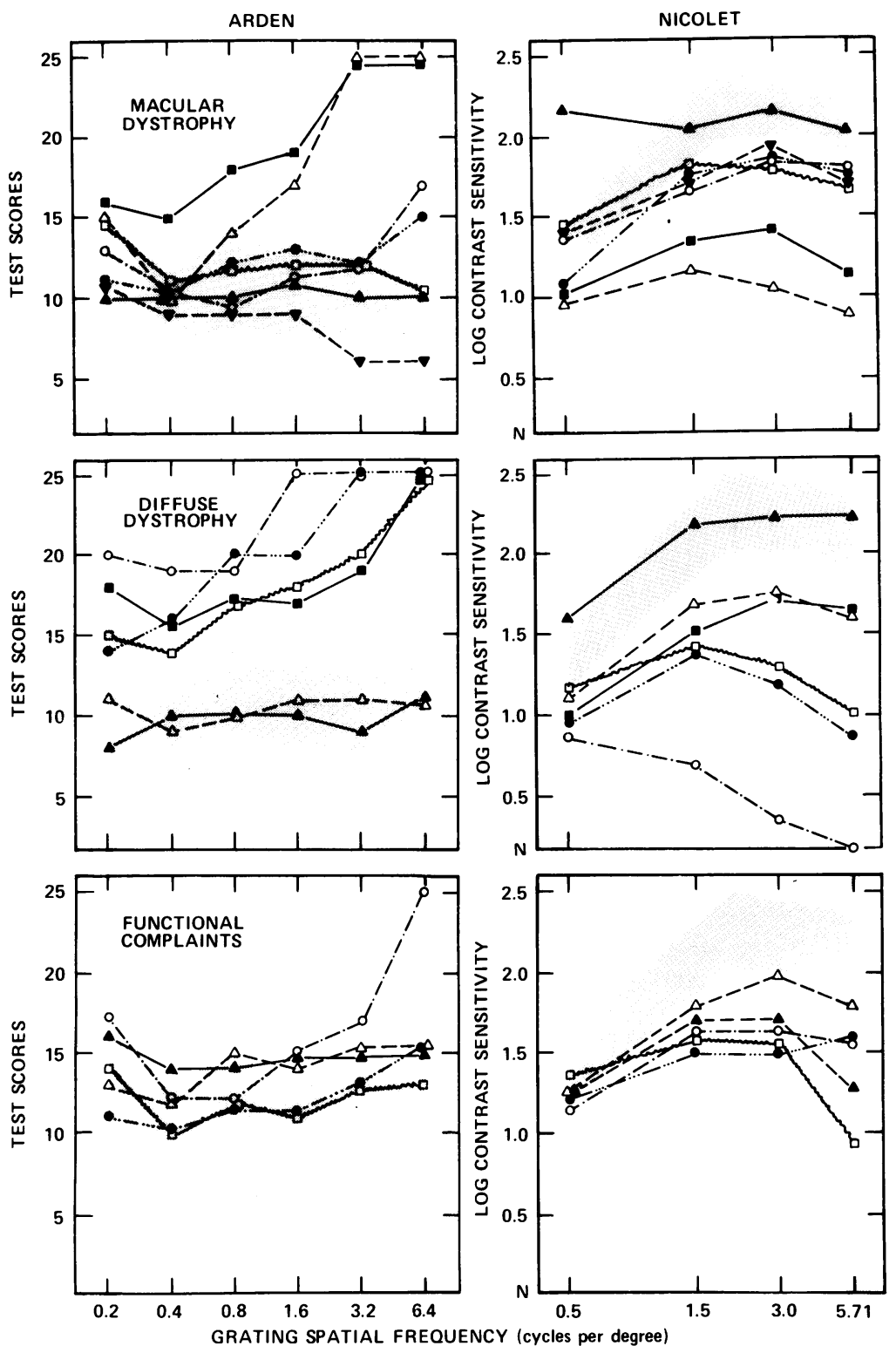

Fig. 5 Comparison between Arden and Nicolet contrast sensitivity scores for eyes with macular dystrophy (top), diffuse dystrophy (middle), and functional complaints (bottom). The range of normal values (mean \pm 2 standard deviations) is shown for each test, but may be high for the Nicolet unit (see text). Each symbol represents a separate patient. Note that good performance is indicated by low Arden scores but high Nicolet scores. contrast sensitivity within the context of visual acuity. Note in Figs. 1-4 that for each disease category even the best recorded total Arden scores increase with acuity in a remarkably linear fashion. This relationship is seen to a lesser degree in the scores for individual gratings. The nature of this covariance is shown better in Fig. 6 . The straight line $C$ represents the best actual scores achieved by our subjects at different levels of acuity: it extends from 2 standard deviations below the $20 / 20$ mean to a score of 150 at a hypothetical acuity of 20/6400*. For comparison line
D represents a theoretical limit beyond which no score can go: it begins near 20/100 where the spatial resolution (visual angle $=5^{\prime}$ ) approximates that of the

*Snellen acuity of 20/20 is defined as the ability to resolve letters of $1^{\prime}$ visual angle; thus, an acuity of $20 / 100$ should be near the optical limit to perceive a grating of $5^{\prime}$ per band, which is close to the spatial frequency of plate 7 (6.4 cpd). Indeed, our data show that some subjects with $20 / 100$ could perceive the plate 7 grating, but none with $20 / 200$ were able to perceive it. By extension $20 / 400$ eyes would miss 3.2 cpd gratings, etc., and subjects with ' $20 / 6400$ ' acuity would be unable to resolve plate $2(0.2 \mathrm{cpd})$ and would therefore miss all the plates. 
Fig. 6 Composite plot of all total Arden scores versus visual acuity. The parallel lines $\mathrm{A}, \mathrm{B}$, and $\mathrm{C}$ intersect 20/20 acuity at the mean score for normal subjects \pm 2 standard deviations. Line D corresponds to the theoretical 'best' scores if reduced acuity affected only the perception of gratings at the limit of resolution. See text.

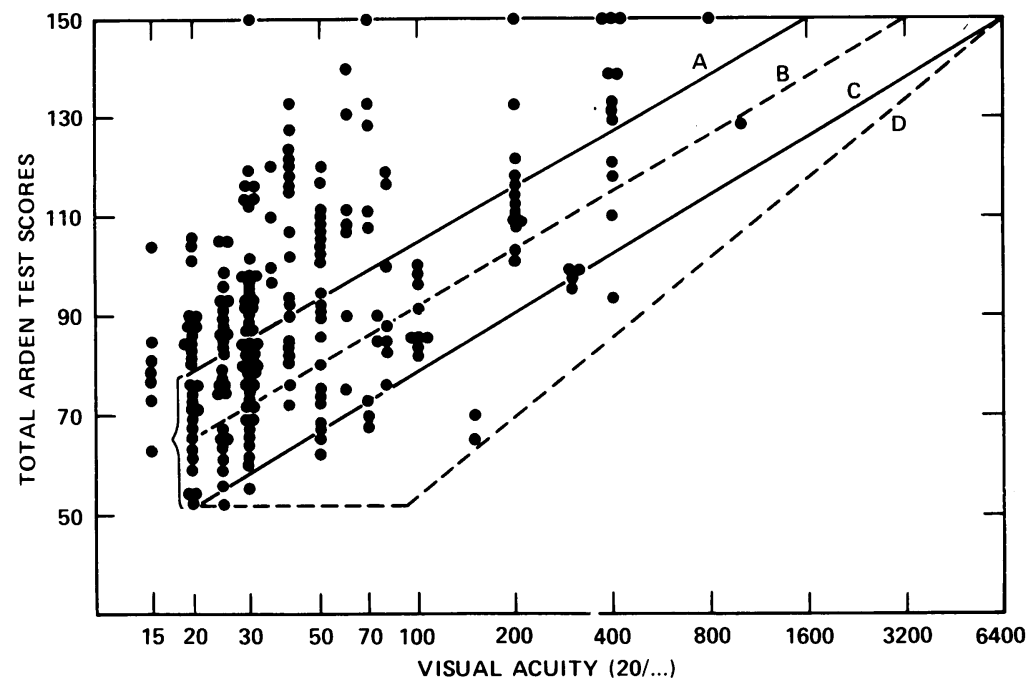

finest Arden grating (6.4 cpd). Any patient with a total score less than line $\mathrm{D}$ must be either anticipating the appearance of the gratings or responding falsely.

Fig. 7 demonstrates that contrast sensitivity falls progressively with decreasing acuity, even for gratings whose spatial frequency is well within the subject's powers of resolution. For example, the 'best' score for $6.4 \mathrm{cpd}$ plate 6 rises with even small losses of resolution (eg., 20/30 and 20/40) that do not come close to compromising perception of the grating. Similarly, when acuity is reduced to $20 / 200$, the scores are elevated for all six plates even though the coarser gratings can still be easily resolved. This suggests that individuals with acuity less than 20/20 may be expected to show elevated Arden scores on the basis of their acuity alone, independently of other pathological concomitants. Only if the contrast deficit exceeds the normal $( \pm 2 S D)$ range that one would expect on the basis of acuity (i.e., the scores are above line A) can we conclude that contrast sensitivity is specifically or preferentially damaged. Contrast sensitivity better than expected on the basis of acuity (i.e., scores below line C) should raise doubts about the reliability or credibility of the subject. Table 1 lists these upper and lower Arden score limits for various levels of visual acuity. Similar graphs and tables could be constructed of course for data from other contrast sensitivity test systems.

This method of analysis has not, to my knowledge, been presented previously, though other studies have skirted some of the issues. Arden' pointed out that mild refractive errors affect only higher spatial frequencies, but he did not report results for a range of acuities. Skalka ${ }^{6}$ plotted some of his data in the same way as ours but did not explore the implications. Campbell and Green ${ }^{13}$ showed effects similar to those in Fig. 7 for a range of different refractive errors, and $\mathrm{Hess}^{14}$ reported different patterns of contrast loss

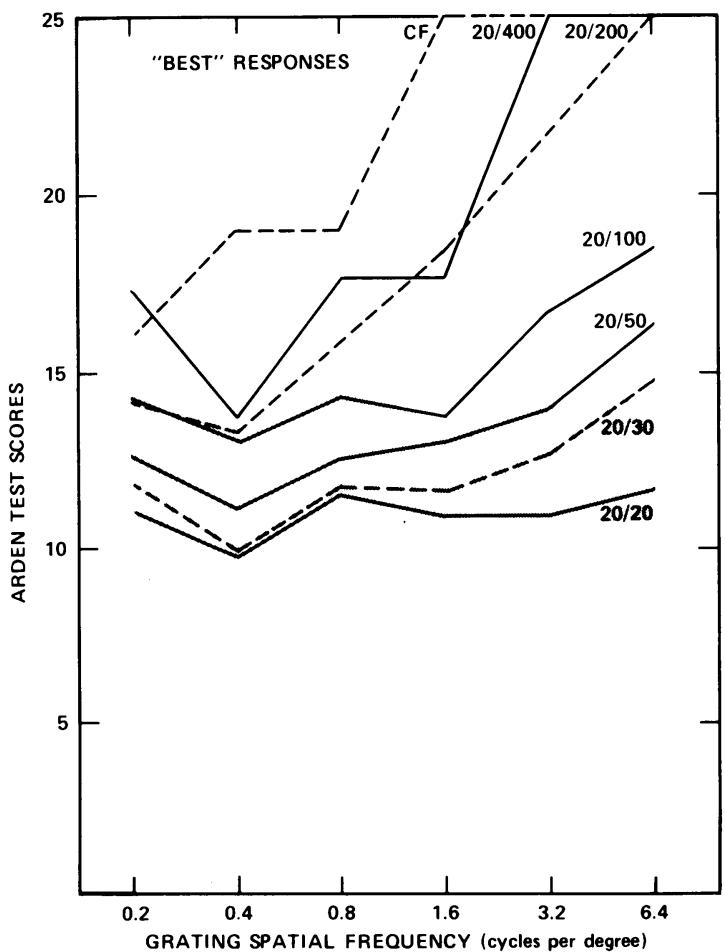

Fig. 7 Individual Arden grating scores averaged from the 'best' eyes at each level of visual acuity (eyes whose total scores fell between lines $\mathrm{A}$ and $\mathrm{C}$ in Fig. 6). The range of values for normal subjects with 20/20 acuity is shown \pm 2 standard deviations. with different causes of poor acuity. But close 
Table 1 Arden norms in relation to visual acuity

\begin{tabular}{llll}
\hline Visual acuity & \multicolumn{3}{l}{ Total Arden scores ${ }^{*}$} \\
\cline { 2 - 4 } & $-2 S D$ & Average & $+2 S D$ \\
\hline $20 / 20$ & 51 & 65 & 78 \\
$20 / 25$ & 55 & 69 & 82 \\
$20 / 30$ & 58 & 72 & 85 \\
$20 / 40$ & 63 & 77 & 90 \\
$20 / 50$ & 67 & 80 & 93 \\
$20 / 70$ & 73 & 86 & 99 \\
$20 / 80$ & 75 & 88 & 101 \\
$20 / 100$ & 79 & 92 & 105 \\
$20 / 200$ & 91 & 104 & 116 \\
$20 / 400$ & 102 & 115 & 127 \\
CF & 118 & 131 & 142 \\
\hline
\end{tabular}

*Data derived from Fig. 6. Scores below -2 SD are 'too good' and should be questioned. Scores above $+2 \mathrm{SD}$ are pathological beyond the usual relationship to reduce acuity. $\mathrm{SD}=$ standard deviation.

comparison with either of these sets of data is difficult because the authors did not report the associated levels of visual acuity. Bodis-Wollner ${ }^{15}$ stated that acuity alone does not predict the pattern of contrast loss. Our data confirm that statement in so far as the more pathological scores are concerned, but our data also suggest that acuity does predict a baseline or 'best' level of contrast sensitivity.

One may criticise our conclusions because the data are derived from subjects with retinal or functional disease rather than from individuals with purely refractive loss of acuity. However, in view of the diversity of diagnoses and the spread of results in this study it does not seem unreasonable to postulate that the very best subjects at each level of acuity had contrast sensitivity scores near the optimum possible (regardless of whether the visual loss was retinal or refractive).

These results are relevant to the clinical application of contrast sensitivity testing. To the extent that a patient's low contrast sensitivity scores reflect only visual acuity, they add little to our diagnostic acumen; in fact they may give a misleading impression of specific pathology. If acuity effects are taken into account, then contrast sensitivity deficits beyond the level accounted for by acuity may have greater significance than previously appreciated.

Supported in part by National Eye Institute Grant EY 01678, the Medical Research Service of the Veterans Administration, and Research to Prevent Blindness, Inc. I thank Dr W J Donovan for helpful comments on the manuscript.

\section{References}

1 Arden GB. The importance of measuring contrast sensitivity in cases of visual disturbance. $\mathrm{Br}$ J Ophthalmol 1978; 62: 198-209.

2 Arden GB, Jacobson JJ. A simple grating test for contrast sensitivity: preliminary results indicate value in screening for glaucoma. Invest Ophthalmol Vis Sci 1978; 17: 23-32.

3 Owsley C, Sekuler R, Siemsen D. Contrast sensitivity throughout adulthood. Vision Res 1983; 23: 689-99.

4 Ginsburg AP. A new contrast sensitivity vision test chart. Am J Optom Physiol Opt 1984; 61: 403-7.

5 Marmor MF. Contrast sensitivity and retinal disease. Ann Ophthalmol 1981; 13: 1069-71.

6 Skalka HW. Comparison of Snellen acuity, VER acuity, and Arden grating scores in macular and optic nerve diseases. $\mathrm{Br} J$ Ophthalmol 1980; 64: 24-9.

7 Wolkstein M, Atkin A, Bodis-Wollner I. Contrast sensitivity in retinal disease. Ophthalmology (Rochester) 1980; 87: 1140-9.

8 Linderg CR, Fishman GA, Anderson RJ, Vasquez V. Contrast sensitivity in retinitis pigmentosa. Br J Ophthalmol 1981; 65: 855-8.

9 Hyvarinen L, Laurinen P, Rovamo J. Contrast sensitivity in evaluation of visual impairment due to macular degeneration and optic nerve lesions. Acta Ophthalmol (Kbh) 1983; 61: 161-70.

10 Kayazawa F, Yamamoto T, Motokazu I. Spatio-temporal modulation transfer function in retinal diseases. Ann Ophthalmol 1983; 15: 662-4.

11 Marmor MF. The electroretinogram in retinitis pigmentosa. Arch Ophthalmol 1979; 97: 1300-4.

12 Ginsberg AP, Cannon MW. Comparison of three methods for rapid determination of threshold contrast sensitivity. Invest Ophthalmol Vis Sci 1983; 24: 798-802.

13 Campbell FW, Green DG. Optical and retinal factors affecting visual resolution. J Physiol (Lond) 1965; 181: 576-93.

14 Hess RF. On the assessment of contrast threshold functions for anomalous vision. Br Orthopt J 1984; 41: 1-13.

15 Bodis-Wollner I. Physiological and clinical aspects of spatialcontrast sensitivity measurements in patients with lesions of the retina and visual pathways. In: Proenza LM, Enoch JM, Jampolsky A, eds. Clinical applications of visual psychophysics. Cambridge: Cambridge University Press, 1981: 42-56.

Accepted for publication 21 November 1985. 